\title{
How to turn the Fast-Track into a Fast-Track: Process integration for evaluation of the quality of Digital Health Applications (DiGAs) on the example of the German Fast-Track Process
}

\author{
Muhammed-Ugur Karagülle \\ Freie Universität Berlin \\ M.U.K@fu-berlin.de \\ Cora Hintze \\ Freie Universität Berlin \\ Cora.Hintze@fu-berlin.de \\ Martin Gersch \\ Freie Universität Berlin \\ Martin.Gersch@fu-berlin.de
}

\author{
Nicolas J. Lehmann \\ Freie Universität Berlin \\ Nicolas.Lehmann@fu-berlin.de \\ Marc-André Juritz \\ Freie Universität Berlin \\ Marc-Andre.Juritz@fu-berlin.de
}

\author{
Agnès Voisard \\ Freie Universität Berlin and \\ Fraunhofer FOKUS \\ Agnes.Voisard@fu-berlin.de
}

\author{
Lea R. Muth \\ Freie Universität Berlin \\ Lea.Muth@fu-berlin.de
}

Lars Stegemann

Freie Universität Berlin

Lars.Stegemann@fu-berlin.de

Joachim W. Fluhr

Charité - Universitätsmedizin Berlin

Joachim.Fluhr@charite.de

\begin{abstract}
In this paper, we address the research question of which integration points in the German Fast-Track process are particularly well suited for the integration of evaluation platforms for digital health applications. For this purpose, possible integration points are first identified and then analyzed with the help of a utility analysis with regard to the posed research question. Finally, a recommendation for action is made based on the results of the conducted utility analysis.
\end{abstract}

\section{Introduction}

As digitalization in medicine continues to grow, digital health applications (in German Digitale Gesundheitsanwendungen - in short DiGA) are expected to gain importance in the future [1]. According to the World Health Organization (WHO), DiGAs have the potential to transform the face of global healthcare [2]. In this context, the quality assessment and evaluation of DiGAs is crucial, as only verified quality can ensure good medical care.

In Germany, the introduction of the Digital Health Act (in German Digitale-Versorgungs-Gesetz - in short $D V G)$ has made prescribable DiGAs eligible for reimbursement since December 19, 2019. For a DiGA to be prescribable, it must be included in the DiGA directory. The costs for prescribable DiGAs are covered by the health insurance companies. In order to be included in the DiGA directory, a DiGA must successfully pass the Fast-Track process of the German Federal Ministry for Drugs and Medicines (BfArM), which is an accreditation process for quality assurance. The DiGAs has also to demonstrate positive care effects in a suitable form, e.g., by additional studies. With the concept of prescription and reimbursement of accredited DiGAs by health insurances, Germany is a pioneer among the OECD countries [1] [4].

Besides the accreditation processes, there are independent evaluation platforms for DiGAs where the evaluation can make detailed statements about the quality of a DiGA and thus positively impacts the accreditation process.

In contrast to accreditation, which in the context of quality assurance only checks whether a condition is fulfilled, an evaluation can make gradual statements about determinable facts. Under the assumption that the evaluation of a quality criterion can be represented as a monotonous, continuous function, a transformation of the evaluation into an accreditation is possible by the discretization of the function in histogram-like stages that logically build on each other along the evaluation dimension of the respective quality criterion, as illustrated in Figure 1

Therefore, we consider the question at which integration point in the German Fast-Track process DiGA evaluation platforms should be integrated to be able to make optimal use of the resulting positive effects?

This paper presents a utility analysis to determine optimal integration points of DiGA evaluation platforms into the DiGA accreditation processes, and taking the 


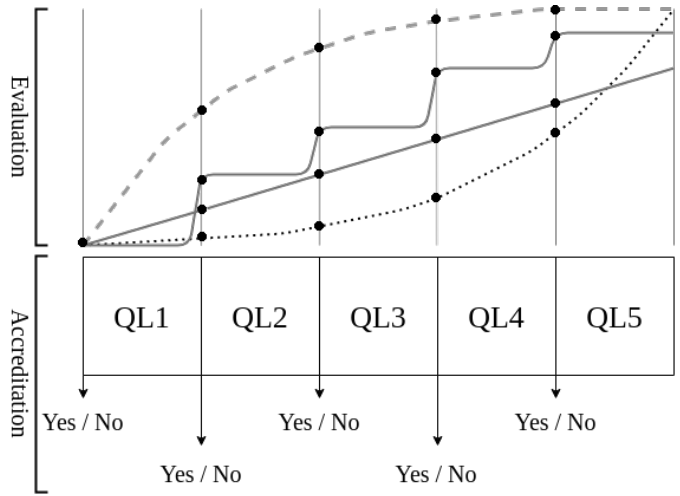

Figure 1. Relationship between a continuous evaluation function and discrete quality levels ( $Q L)$ derived by decision boundaries for accreditation.

German Fast-Track [5] process as an example. First, the used criteria, scales, and perspectives of the used utility analysis are introduced. Then, possible integration points in the German Fast-Track process are identified. Subsequently, the criteria are evaluated in the form of an author discussion for each identified integration point. Afterward, different perspectives are presented in the context of the utility analysis. Finally, the results of the utility analysis are compared, and a recommendation for the integration of DiGA evaluation platforms into the German Fast-Track process is formulated.

This paper is structured as follows. Section 2 provides an overview of related work focused on the accreditation and evaluation approaches of DiGAs, including a brief explanation of the German Fast-Track process, which is used as a model reference. Furthermore, approaches for the evaluation of process integrations are presented. In Section 3, the methods used to identify and evaluate appropriate integration points of DiGA evaluation platforms into DiGA accreditation processes are presented. Integration points for DiGA evaluation platforms into the German Fast-Track process are identified in Section 4. Section 5 discusses and concludes the evaluation of the selected criteria for each integration point. In Section 6, we draw our conclusion.

\section{Related work}

\subsection{Accreditation of DiGAs for the purpose of quality assurance}

The Food and Drug Administration (FDA) accreditation process classifies DiGAs into three risk classes (low, middle, high) with associated regulatory requirements based on the underlying utility. The FDA verifies that these requirements have been fully validated and implemented. Depending on the class, scientific evidence of the DiGA's safety and effectiveness may be required.

In comparison, the German Digital Health Care Act (Digitale-Versorgung-Gesetz) and the Digital Health Applications Ordinance (Digitale Gesundheitsanwendungen-Verordnung) specify that for a DiGA to be included in the DiGA directory, it must first undergo a review by the Federal Institute for Drugs and Medical Devices (Bundesinstitut für Arzneimittel und Medizinprodukte - BfArM). This review is called the German Fast-Track process, which is illustrated in Figure 2 During the Fast-Track process,

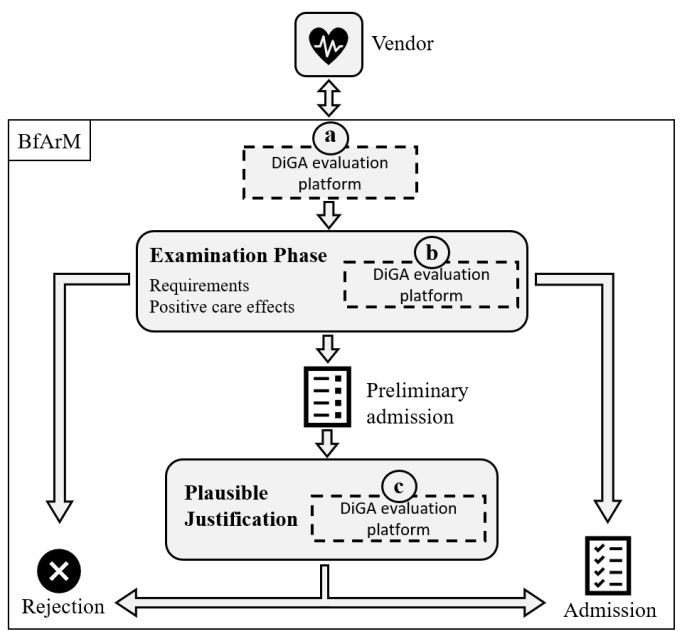

Figure 2. Integration points for $\mathbf{m H e a l t h}$ evaluation platforms in the German Fast-Track process [5].

the BfArM first reviews for at least three months (a) the specified requirements for safety, functionality, quality, interoperability, data protection, and data security are implemented. In Step (b), the DiGA has to proof positive care effects or medical benefit in terms of procedural and structural improvement. Three possible cases may arise within this review. Either the DiGA already fulfills all criteria and can be included directly in the DiGA directory, or if this is not the case, the DiGA can be rejected directly. Furthermore, in case of doubt regarding the positive care effect, the BfArM retains the right to include the DiGA only provisionally in the DiGA directory and test it for a positive care effect in a 12-month trial phase (C). After that, the DiGA is either added to the DiGA directory or rejected. If the DiGA is included in the DiGA directory, physicians and psychotherapists can prescribe it to their patients by prescription, and a statutory health insurance company covers the costs. 


\subsection{Evaluation of DiGAs for a differentiated assessment of their quality}

To date, there are several different approaches to determine the quality of a DiGA. One widely used approach is the determination of the quality of DiGAs by answering questionnaires. Besides this widely used approach, other approaches provide guidelines or define rules, or heuristics, that DiGA vendors should follow. In [6], the authors provide a broad overview of methodological and practical approaches to evaluate the quality of DiGAs.

In [7], the authors present eight principles that should lead to high quality medical websites (or applications). The presented HONcode includes the principles Authoritative, Complementarity, Privacy, Attribution, Justifiability, Transparency, Financial disclosure, and Advertising policy. Medical websites (or applications) that successfully undergo voluntary audits by Health On the Net Foundation (HON) receive a quality seal to help physicians and patients navigate the digital health market.

In [8] and [9], the authors present a questionnaire to determine the quality of a DiGA in two versions. The Medication Adherence Rating Scale (MARS) is a questionnaire aimed at physicians that determines the quality of a DiGA in five domains. The User Medication Adherence Rating Scale (uMARS) is based on the Medication Adherence Rating Scale, but it addresses patients or users of DiGAs instead of physicians.

In [10], the Fraunhofer FOKUS presents a catalog of questions suitable for the evaluation of the quality of DiGAs. The questions cover a wide range of issues related to the topics of medical content, ethics, security, technical features, and privacy. The AppKri question catalog does not address a specific target group and does not serve as a questionnaire for evaluating the quality of DiGAs on its own but as a toolbox for creating such questionnaires.

In [11], [12], and [13], the authors present an expert-based multidisciplinary approach to the evaluation of DiGAs. The approach follows the idea of a multidisciplinary evaluation with experts from the four domains of medical Content, Usability, Security, and Law, of DiGAs. The quality evaluation of DiGAs is performed with the CUSL score, which also can be used as a quality seal, comparable to the HON quality seal. Furthermore, the concept of an DiGA evaluation platform is presented, which provides quality information of DiGAs with a structured taxonomy to patients, physicians, and health insurances.

Beyond the approaches mentioned above to determine the quality of DiGAs, some platforms concretely implement the quality evaluation of DiGAs.

Weisse Liste [14] is a German DiGA evaluation platform operated by a subsidiary of Bertelsmann Verlag. The platform enables physicians and patients to search for DiGAs and provides detailed information about the quality of DiGAs through voluntarily produced quality reports by DiGA vendors. Furthermore, the platform offers additional services, such as a physician search or functions for finding hospitals and care homes.

KVAppRadar [15] by Central Institute for the Provision of Health Care by Statutory Health Insurance Physicians in the Federal Republic of Germany Foundation under civil law (Zentralinstitut für die kassenärztliche Versorgung in der Bundesrepublik Deutschland Stiftung des bürgerlichen Rechts) is a German DiGA evaluation platform that provides DiGA evaluation data only for health insurances and physicians. As core functions, the platform enables physicians a structured discussion and evaluation of DiGAs and the communication of the involved stakeholders.

ORCHA [16] is a British evaluation platform aimed at patients, physicians, and DiGA vendors. The platform offers DiGA vendors the opportunity to evaluate their DiGAs independently by medical experts. Reviewed DiGAs are presented on the ORCHA platform with detailed information about their quality. Physicians and patients can search for and inform themselves about DiGAs via the platform. Beyond the one-time evaluation of a DiGA, the platform ensures a continuous quality evaluation of all tested DiGAs.

\subsection{Process integration}

The evaluation of processes within the context of process management is required in order to be able to identify, evaluate and thus compare possible enhancements. The accreditation often used for this purpose can be of too limited precision.

Therefore, the author of [17] extends the Software Process Improvement and Capability Determination (SPICE) based accreditation by an evaluation. SPICE describes an overall process based on process attributes and maturity levels. These represent the performance of the individual processes, from which follows process evaluation on a scale of 0 (incomplete) to 5 (optimizing).

The author points out that only the combination of quantitative and qualitative evaluation ensures a successful overall assessment of the processes. Performance deficits can be identified through the quantitative assessment, and possible starting points for improvements can be derived from the qualitative 
evaluation.

Often, pure accreditation with subsequent or preliminary evaluation is still too static, as different stakeholders may have different sets of requirements.

To include these requirements as well, the authors of [18] present the Business Process Blueprinting (BPBP) method, which serves as an integrated method for the targeted analysis, planning, design, and control of (value-added) processes with distinctive (service) characteristics. The method is based on the fundamental challenge that elementary components of information management often do not have a corresponding process model (event-driven process chains (EPC)). Therefore, [18] opens another opportunity to identify weak points in processes and closes them from the perspective of information and service management. The method looks at the structural design of a process at different levels of its activity, Interaction, Visibility, Internal Interaction, Oder Penetration, and Implementation. These serve as the foundation for an evaluation system (measurement system), which measures internal efficiency and its decisive influences and thus makes them measurable.

In [21], the criteria Time, Costs, Quality, Capacity, Flexibility, Integration, and Complexity for the evaluation of process performance are introduced.

A standardized and thus proven utility analysis does not exist for the evaluation and accreditation of mHealth applications. Inspired by the authors' identification of weaknesses of [17] and the use of different perspectives, proposed in [18], as well as the criteria selection of [21], our proposed methodology has emerged.

\section{Methodology}

\subsection{Identification of integration points}

An author discussion with advantages and disadvantages along the German Fast-Track process is conducted to identify weaknesses as they can be considered to be potential points of integration according to [17]. Furthermore, for this purpose, the systematic structure of the German Fast-Track process from different levels, as presented in [18], is analyzed in order to evaluate the identified weaknesses later.

\subsection{Evaluation of integration points}

A utility analysis [19] [20] is used to assess the effectiveness of the integration of evaluation platforms at the possible identified positions of the integration points. In the utility analysis, the sums of the weighted criteria of the different integration points are compared with each other under consideration of different perspectives in order to be able to derive a general recommendation for action for the integration of evaluation platforms into the German Fast-Track process.

Table 1. Criteria catalogue.

\begin{tabular}{|l|l|}
\hline Criteria & Description \\
\hline Time & $\begin{array}{l}\text { - Process duration } \\
\text { - Idle, control, and lead time }\end{array}$ \\
\hline Costs & $\begin{array}{l}\text { - Operating costs } \\
\text { - Error costs }\end{array}$ \\
\hline $\begin{array}{l}\text { Inter- } \\
\text { operability }\end{array}$ & $\begin{array}{l}\text { - Standardization and automatization } \\
\text { - Process flow and accessibility }\end{array}$ \\
\hline Capacity & $\begin{array}{l}\text { - Throughput and bottlenecks } \\
\text { - Workload and performance }\end{array}$ \\
\hline Flexibility & $\begin{array}{l}\text { - Adaptability and ability to handle } \\
\text { changed environmental conditions }\end{array}$ \\
\hline Complexity & $\begin{array}{l}\text { - Process structure } \\
\text { - Degree of interaction }\end{array}$ \\
\hline
\end{tabular}

We have selected criteria from the criteria catalog that are relevant from an international perspective [21]. The selection of criteria is presented in Table 1 The first criterion is Time since, according to [22], DiGAs need to be prescribable quickly, as patients are likely to use DiGAs regardless of clinical support or guidance, and this could cause health authorities and providers to fall further behind. There is an interest in saving healthcare Costs [23], and thus resources through the use of digital solutions, which can be assisted by proper Interoperability of systems [24]. It could be observed whether the integration of the DiGA evaluation platform leads to an increase in Capacity, i.e., the number of DiGAs to be evaluated increases. It is important to include Flexibility as an evaluation criterion, frequently disjointed and static solutions cannot be adequately adapted to environmental changes or the concerns of stakeholders and lead to problems [22]. The extent to which platforms for evaluating the quality of DiGAs can be integrated into the information flow and workflow of the processes is being investigated. The goal is to achieve increased automatization. Relevant for successful integration is the accessibility and granularity of the information needed by the platforms for evaluation. The final criterion is Complexity. Reducing complexity could result in healthcare providers being able to perform better [23].

The scale used for the utility analysis consists of the five nominal values very poor, poor, medium, good, and very good. The values are mapped against the discrete values 1 for very poor, 2 for poor, 3 for medium, 4 for good, and 5 for very good for the later calculation of the total values from which the ranks of the integration 
points are derived. Due to the lack of data for the present consideration, we evaluate certain criteria on the basis of a relative comparison between the ranks of the integration points. The lowest rank is rated as poor, the medium rank as medium and the highest rank as good.

The weighting scale uses the five nominal values very low, low, medium, high and very high. The weights are mapped against the discrete values $0 \%$ for very low, $25 \%$ for low, $50 \%$ for medium, $75 \%$ for high and $100 \%$ for very high. Since the sum of the weights can reach more than $100 \%$, the values are normalized to $100 \%$ for the comparison of the perspectives.

The implementation of the utility analysis is based on an author discussion of the individual criteria for each integration point. Finally, the evaluation of the criteria is compared by different perspectives, and a general recommendation for action is derived.

\section{Identification of integration points of DiGA evaluation platforms into the German Fast-Track process}

A systematic inspection of the German Fast-Track process results in five formally possible integration points. The first possible integration point is located before the submission of the application for approval of a DiGA by the DiGA vendor. The second possible integration point is during the examination of the requirements regarding the quality criteria and the proof of the positive care effects of the DiGA by the BfArM. The third possible integration point is after the accreditation of the quality criteria and the proof of positive care effects. The fourth possible integration point is during the Plausible Justification Phase, in which the proof of positive care effects is verified. The fifth and final possible integration point is located after the Plausible Justification Phase.

Integration of evaluation platforms into the Fast-Track process can only impact the parts of the process where accreditation of quality criteria takes place. In the entire Fast-Track process, only during the Examination Phase an examination of quality criteria takes place. Therefore, we limit our consideration of integration points to the following three of the five possible integration points.

\section{Preliminary Check (prior to the Examination Phase, (a) in Figure 2).}

2. Examination Phase (during the Examination Phase, (b) in Figure 2).

3. Plausible Justification Phase (after the Examination Phase, (C) in Figure 2).

\section{Evaluation of integration points of DiGA evaluation platforms into the German Fast-Track process}

The following discussion and argumentative considerations represent the authors' opinion after previous communication with the BfArM. The assignment of the value score is exclusively due to the authors, as no statement could be received from the BfArM in this regard. The following criteria are discussed holistically but evaluated based on a worst-case scenario since this is exclusively relevant for evaluating a possible integration point. Due to the lack of data for the present observation, we evaluate some criteria using a relative comparison between the ranks of integration points.

\subsection{Preliminary Check}

Time: The integration of an evaluation platform in the Preliminary Check, before the Examination Phase, results in more time passing until the Examination Phase begins. In the overall consideration of downstream phases, the Examination Phase benefits from the results of the evaluation platform, and the upstream criteria evaluation can save time. Since only positive care effects are examined in the remaining phases, no further time savings are expected. Assuming that positive care effects can be demonstrated in the examination phase following the Preliminary Check, time can potentially be saved to the extent of the total process duration of up to 3 months. If positive care effects cannot be demonstrated to a sufficient extent in the Examination Phase, the total process duration can run up to 15 months (Examination Phase + Plausible Justification Phase). In this case, only time to the extent of up to 3 months of the Examination Phase can be saved. Assuming that no Plausible Justification Phase is required, we rate the criterion Time for the integration point Preliminary Check as poor since a maximum time saving is only $20 \%$ (3 of 15 months). With the Plausible Justification Phase's connection, the potential savings increase to up to 15 months (Examination Phase + Plausible Justification Phase).

Costs: For exact costs estimation, no detailed information about the actual costs of the BfArM is available. Therefore, a proportional ratio of customer costs to BfArM in-house costs is assumed. The fees estimated by the customers can be obtained from the BfArM's guide for the Fast-Track process [3]. For the evaluation of the cost criterion, the worst-case is considered from the perspective of the DiGA vendor, 
and these are in the longest possible case up to 35700 EUR. The savings potential of integrating an evaluation platform is assumed for the best-case scenario and amounts up to 11100 EUR, as money can only be saved in the Examination Phase by integrating an evaluation platform. As a result, there is a total savings potential of up to $31 \%$. Due to the selected evaluation scale, which is divided into $20 \%$ steps, the savings potential of the criterion Costs for the integration point Preliminary Check is rated as poor.

Capacity: With the integration of an evaluation platform into the Preliminary Check, in the best-case, the complete criteria check within the Examination Phase can be omitted. Furthermore, by placing the evaluation platform upstream of examining positive care effects, the condition is achieved that the evaluation platform exclusively examines quality criteria and the BfArM exclusively examines positive care effects. An increase in performance for the overall process can be achieved through the specialization of the evaluation platform with the evaluation of the criteria and the specialization of the BfArM with the examination of the positive care effects. Therefore, we rate the criterion Capacity by means of relative comparison between the integration points, the integration of an evaluation platform in the Preliminary Check as good.

Flexibility: The criterion Flexibility receives in comparison between the integration points the relative rating medium since maximum flexibility is given in this phase because all possible changes in environmental conditions can be counteracted in the Examination Phase without consequences due to the knowledge gained in the Preliminary Phase.

Interoperability: The integration of an evaluation platform in the Preliminary Check enables a high degree of interoperability since the results of the evaluation platform are transferred at once, directly and entirely to the accreditation process. In relative comparison between the integration points, we rate the criterion Interoperability of the integration point Preliminary Check as good.

Complexity: By integrating an evaluation platform into the Preliminary Check, the overall process structure of the Fast-Track process is linearized and thereby simplified. The knowledge of the evaluation platform is guided linearly through each further process step, which means that the examination of the quality criteria in the Examination Phase can be omitted. Therefore, we rate the criterion Complexity for the integration point Preliminary Check integration point as good.

\subsection{Examination Phase}

Time: Assuming that the processes can be fully integrated and positive synergy effects occur, the Fast-Track process can only benefit from the evaluation platform results after they have been generated. Therefore, the potential time savings at this point are lower compared to the integration of evaluation platforms in the Preliminary Check. The speed with which the evaluation platform results are generated determines the actual time savings. Assuming that positive care effects have already been successfully demonstrated in the Examination Phase, time can potentially be saved to the extent of the total process duration of 3 months subtracted by the time needed for the evaluation platforms to generate results. If positive care effects cannot be demonstrated to a sufficient extent in the Examination Phase, or if this is clear in advance, the total process duration can be up to 15 months. In this case, only time within the Examination Phase can be saved to the extent of up to 3 months subtracted by the time needed by the evaluation platforms to generate results. Based on the present observation, we rate the criterion Time for the Examination Phase integration point as very poor, since in the worst-case scenario, the time required by the evaluation platforms to generate results is a maximum of 3 months and thus no time savings ( 0 of 3 months) are achieved.

Under the hypothetical assumption that evaluation platforms can also demonstrate positive care effects, the potential savings would be significantly higher at up to 15 months subtracted by the time needed for evaluation platforms to generate results.

Costs: For exact cost estimation, no detailed information about the actual costs of the BfArM is available. Therefore, a proportional ratio of customer costs to BfArM in-house costs is assumed. The fees estimated by the customer can be obtained from the BfArM's guide for the Fast-Track process [3]. For the evaluation of the criterion, the worst-case is considered from the perspective of the DiGA vendor, and these are in the longest possible case up to 35700 EUR. The savings potential of integrating an evaluation platform is assumed for the best-case scenario and amounts to up to 11100 EUR, as costs can only be saved in the Examination Phase by integrating an evaluation platform. As a result, there is a total savings potential of up to $31 \%$. Due to the selected evaluation scale, which is divided into $20 \%$ steps, the savings potential of the 
criterion Costs for the integration point Examination Phase is rated as poor.

Capacity: By integrating an evaluation platform into the Examination Phase, a part of the accreditation of the quality criteria is eliminated since the evaluation platform can cover it during the examination. Therefore, we rate the criterion Capacity for the integration point Examination Phase due to the released capacities by means of relative comparison between the integration points as medium.

Flexibility: Since the evaluation platform has more interaction points in the Examination Phase than in the Preliminary Check, more parts are affected by changes. This fact reduces the flexibility of the Fast-Track process to handle changes in environmental conditions or process structure compared to the Preliminary Check. The flexibility in the Examination Phase is lower in direct comparison to the Plausible Justification Phase since it has no interaction points. Therefore, we rate the criterion Flexibility for the integration point Examination Phase by means of relative comparison between the integration points as poor.

Interoperability: The need for interoperability is exceptionally high in the Examination Phase because the accreditation process and the evaluation platform have a high need for interaction in their examination of the quality criteria due to the parallel process design. Therefore, we rate the criterion Interoperability for the integration point Examination Phase by means of relative comparison between the integration points as medium.

Complexity: The integration of an evaluation platform in the Examination Phase leads to an interaction-related complexity of the process structure due to the parallel process of the accreditation of the quality criteria and the evaluation of the positive care effects. By integrating the evaluation platform into the Examination Phase, the sub-process of accreditation of the quality criteria can be replaced by the evaluation platform, which increases the complexity. The integration of the additional platform implies an increased communication effort and a corresponding higher interoperability. In the relative comparison between the possible integration points, we therefore rate the criterion Complexity in the Examination Phase as poor.

\subsection{Plausibility Justification Phase}

Time: The integration of an evaluation platform into the Plausibility Justification Phase does not influence the duration of the Fast-Track process since evaluation platforms are usually not suitable for evaluating positive care effects that are examined in the Plausibility Justification Phase. Based on the present observation, we rate the criterion Time for the integration point Plausibility Justification Phase as very poor, since no time savings are achieved in the worst-case scenario ( 0 of 12 months).

Under the hypothetical assumption that evaluation platforms could also demonstrate positive care effects, potential savings equal to the total process duration of up to 12 months would be made possible.

Costs: For exact cost estimation, no detailed information about the actual costs of the BfArM is available. Therefore, a proportional ratio of customer costs to BfArM in-house costs is assumed. The fees estimated by the customer can be obtained from the BfArM's guide for the Fast-Track process [3]. For the evaluation of the criterion, the worst-case is considered from the perspective of the DiGA vendor, and these are in the longest possible case up to 35700 EUR. The savings potential of integrating an evaluation platform is assumed for the best-case scenario and amounts to 0 EUR, as the integration of the evaluation platform does not influence the Examination Phase. As a result, there is a total savings potential of $0 \%$. Therefore, we rate the criterion Costs for the integration point Plausibility Justification Phase as very poor.

Capacity: When an evaluation platform is integrated into the Plausibility Justification Phase, interactions are unnecessary due to the lack of need for quality criteria evaluations. Furthermore, integration at this point has no impact on throughput or generates any changes in the performance of the overall process. Therefore, in relative comparison between the integration points, we rate the criterion Capacity for the integration point Plausibility Justification Phase as poor.

Flexibility: Since the evaluation platform has little or no interaction points in the Plausible Justification Phase, no parts of the Fast-Track process are affected by environmental changes. This fact increases the flexibility of the Fast-Track process to handle changes in environmental conditions or process structure compared to the Preliminary Check. Therefore, the flexibility in the Plausible Justification Phase is higher than in any other integration point. Hence, we rate the 
Table 2. Utility analysis - Perspective: Integration.

\begin{tabular}{|l|l|ll|ll|ll|}
\hline \multirow{2}{*}{ Criteria } & \multirow{2}{*}{ Weighting } & \multicolumn{2}{|c|}{ Preliminary Check } & \multicolumn{2}{|l|}{ Examination Phase } & \multicolumn{2}{l|}{ Plausible Justification Phase } \\
\cline { 2 - 8 } & Rating & Score & Rating & Score & Rating & Score \\
\hline Time & $50 \%$ (medium) & 2 (poor) & 1.00 & 1 (very poor) & 0.50 & 1 (very poor) & 0.50 \\
Costs & $25 \%$ (low) & 2 (poor) & 0.50 & 2 (poor) & 0.50 & 1 (very poor) & 0.25 \\
Capacity & $50 \%$ (medium) & 4 (good) & 2.00 & 3 (medium) & 1.50 & 2 (poor) & 1.00 \\
Flexibility & $25 \%$ (low) & 3 (medium) & 0.75 & 2 (poor) & 0.50 & 4 (good) & 1.00 \\
Interoperability & $100 \%$ (very high) & 4 (good) & 4.00 & 3 (medium) & 3.00 & 2 (poor) & 2.00 \\
Complexity & $25 \%$ (low) & 4 (good) & 1.00 & 4 (good) & 1.00 & 3 (medium) & 0.75 \\
\hline$\sum$ & $275 \%$ & 19 & 9.25 & 15 & 7.00 & 13 & 5.50 \\
\hline Normalized & $\mathbf{1 0 0 \%}$ & & $\mathbf{3 . 3 6}$ & & $\mathbf{2 . 5 5}$ & & $\mathbf{2 . 0 0}$ \\
\hline
\end{tabular}

criterion Flexibility for the integration point Plausible Justification Phase by means of relative comparison between the integration points as good.

Interoperability: The need for interoperability is low in the Plausible Justification Phase because the examination of positive care effects does not need interaction with an evaluation platform, as no quality criteria are evaluated. Furthermore, integrating an evaluation platform in this integration point has no impact on the process flow and, therefore, no need for standardization or automatization. Therefore, we rate the criterion Interoperability for the integration point Plausible Justification Phase by means of relative comparison between the integration points as poor.

Complexity: The integration of an evaluation platform into the Plausible Justification Phase creates a simple process structure since the evaluation of the quality criteria takes place linearly downstream of the accreditation of the quality criteria in the Examination Phase and an evaluation platform can therefore not make a positive contribution to the overall process. Since, in contrast to the integration of an evaluation platform into the Preliminary Check, no positive effects can be achieved in the Examination Phase. Therefore, we rate the criterion Complexity for the integration point Plausible Justification Phase by means of relative comparison between the integration points as medium.

\section{Perspectives}

For the utility analysis, we consider different integration perspectives. The Integration, Efficiency, and Scalability perspectives are based on the assumption of the criticisms by the German National Association of Health Insurers that the Fast-Track process is inadequately designed to adequately guarantee the requirements for the factors Use-value, Quality, and Efficiency, as outlined in [25]. In addition, we introduce the Balance perspective, in which the criteria are weighted equally (very high $\widehat{=} 100 \%$ ) to provide a baseline comparison between all criteria. Each perspective, except the Balance perspective, weights the criteria differently to reflect the selected focus.

\subsection{Perspective: Integration}

First, we take a look at the integration perspective. The criterion Interoperability is the dominant factor, with a rating of very high (very high $\widehat{=} 100 \%$ ). The criteria Time and Capacity have a higher relevance with the rating medium (medium $\widehat{=} 50 \%$ ) than the remaining criteria, which are rated low (low $\widehat{=} 25 \%$ ). The results of this perspective are illustrated in Figure 2

\subsection{Perspective: Balance}

Second, we define the Balance view in which all evaluation criteria are equally weighted. The resulting ranking does not differ from the ranking of the Integration perspective, despite a slight change in the score values, as resented in Table 3 .

\subsection{Perspective: Efficiency}

Third, we adopt the Efficiency perspective, which weights the two criteria of costs (very high $\widehat{=} 100 \%$ ) and time (high $\widehat{=} 100 \%$ ) strongest. This weighting expresses our focus on reducing costs and shortening the application evaluation process. We assign medium importance to the criteria Interoperability (medium $\widehat{=}$ $50 \%)$ in this consideration. The remaining criteria are weighted (low $\widehat{=} 25 \%$ ).

The resulting ranking does not differ from the ranking of the Integration perspective despite a change in the values, as resented in Table 3 
Table 3. Ranking of integration points according to perspectives: Integration, Balance, Efficiency, and Scalability.

\begin{tabular}{|c|cc|ccccccc|}
\hline \multirow{2}{*}{ Integration Point } & \multicolumn{7}{|c|}{ Perspective } \\
\cline { 2 - 9 } & \multicolumn{2}{|c|}{ Integration } & \multicolumn{2}{|c|}{ Balance } & Efficiency & \multicolumn{2}{c|}{ Scalability } \\
\cline { 2 - 9 } & Rank & Score & Rank & Score & Rank & Score & Rank & Score \\
\hline Preliminary Check & 1 & 3.36 & 1 & 6.91 & 1 & 3.18 & 1 & 4.09 \\
Examination Phase & 2 & 2.54 & 2 & 5.45 & 2 & 2.45 & 2 & 3.27 \\
Plausible Justification Phase & 3 & 2.00 & 3 & 4.73 & 3 & 1.91 & 3 & 3.00 \\
\hline
\end{tabular}

\subsection{Perspective: Scalability}

Fourth, we set the focus on the Scalability perspective. The criterion capacity has the highest relevance and is therefore weighted as very high (very high $\widehat{=} 100 \%$ ). The criteria complexity and flexibility also have high relevance in this perspective and are therefore rated high (high $\widehat{=} 75 \%$ ). All other criteria have a low influence (low $\widehat{=} 25 \%$ ).

The resulting ranking does not differ from the ranking of the Integration perspective despite a change in the values, as resented in Table 3 .

\section{Conclusion}

\subsection{Discussion of results}

As a result of the utility analysis conducted to find the best possible integration point for DiGA evaluation platforms, we recommend the integration point Preliminary Check due to the overall positive effects on Time, Costs, Capacity, Interoperability, and Complexity as represented in Table 3

The evaluation platform can act as a gatekeeper in this integration point and optimally save work and costs over the entire process since the evaluation results are available before the Fast-Track process, and therefore the scope of the accreditation measures can be derived.

Regardless of the perspective taken, the Preliminary Check represents the best integration point for integrating DiGA evaluation platforms into the German Fast-Track process. The Examination Phase ranks second before the Plausible Justification Phase on the third rank. As this integration point reaches the highest score, it is preferred over the other identified integration points.

Since the utility analysis is based on estimates in some places, values may deviate. Although the estimates are made to the best of the authors' knowledge and belief, they are naturally subject to fuzziness. Also problematic is the relative comparison of some criteria of the integration points with each other since no absolute values can be determined for an exact comparison. Furthermore, the mapping of nominal values to numerical values is problematic. Nevertheless, we believe that we can make a generally valid statement despite the weaknesses of the used model, since no other adequate method of investigation is available.

\subsection{Limitations}

Despite the theoretical assumption that an optimal integration point for DiGA evaluation platforms is known, technical and structural problems of data integration are still an obstacle today for a concrete implementation of a DiGA evaluation platform in the German Fast-Track process. A special role is played by missing or not yet implemented technical, semantical, and syntactical standards [1] [26].

In addition, the investigation carried out in this paper assumes that the integration of DiGA evaluation platforms into DiGA accreditation processes, using the German Fast-Track process as an example, produces exclusively positive effects. The authors' assumption is based on the fact that the integration of such evaluation platforms into accreditation processes is only helpful if they produce positive effects.

\subsection{Future work}

This paper approach only analyzes the German Fast-Track process. A repetition of this approach for other DiGA accreditation processes, such as the one of the FDA, would be desirable in order to be able to make more generalizable statements. Furthermore, the conceptual considerations of this paper should be evaluated by integrating different concrete DiGA evaluation platforms. Finally, it should be noted that the underlying assumption of this paper, that the integration of DiGA evaluation platforms into DiGA accreditation processes generates only positive effects, should be reviewed, especially concerning the question of whether adverse effects can also occur as a result of the integration. 


\section{References}

[1] S. Gerke, A. D. Stern, and T. Minssen, Germany's digital health reforms in the COVID-19 era: lessons and opportunities for other countries, Nature, NPJ digital medicine, vol. 3, p. 94, 2020.

[2] WHO Global Observatory for eHealth, mHealth: new horizons for health through mobile technologies: second global survey on eHealth, Global observatory for eHealth Series, 3: World Health Organization, 2011.

[3] Federal Institute for Drugs and Medical Devices Germany (Bundesinstitut für Arzneimittel und Medizinprodukte (BfArM)), Das Fast-Track-Verfahren für digitale Gesundheitsanwendungen (DiGA) nach $\$$ $139 e S G B V$, Federal Institute for Drugs and Medical Devices Germany (Bundesinstitut für Arzneimittel und Medizinprodukte (BfArM)), 2020. (in German)

[4] U.-V. Albrecht, Chances and Risks of Mobile Health Apps (CHARISMHA), Hanover Medical School, In: U.-V. Albrecht (Ed.) Rationale, pp. 2-5, 2016.

[5] Federal Institute for Drugs and Medical Devices (Bundesinstitut für Arzneimittel und Medizinprodukte (BfArM)), The Fast-Track Process for Digital Health Applications (DiGA) according to § 139e SGB V, 2020.

[6] P. Azad-Khaneghah, N. Neubauer, A. Miguel Cruz, and L. Liu, Mobile health app usability and quality rating scales: a systematic review, Taylor \& Francis, Disability and Rehabilitation: Assistive Technology, pp.1-10, 2020.

[7] C. Boyer, V. Baujard, and A. Geissbuhler, Evolution of health web certification through the HONcode experience, IOS Press, Studies in health technology and informatics, vol. 169, pp. 53-57, 2011.

[8] S. R. Stoyanov, L. Hides, D. J. Kavanagh, O. Zelenko, D. Tjondronegoro, and M. Mani, Mobile app rating scale: a new tool for assessing the quality of health mobile apps, JMIR Publications Inc., JMIR mHealth and uHealth, vol. 3, num. 1, p. e27, 2015.

[9] S. R. Stoyanov, L. Hides, D. J. Kavanagh, H. Wilson, Development and validation of the user version of the Mobile Application Rating Scale (uMARS), JMIR Publications, JMIR mHealth and uHealth, vol. 4, num. 2, p. e5849, 2016

[10] Fraunhofer Fokus, Telehealth - APPKRI, 2018, https://www.innovationszentrum-telehealth.de/go/appkri. (Online; Accessed September 21, 2021) (in German)

[11] N. J. Lehmann, M.-U. Karagülle, D. Kmiotek, F. Spielmann, B. George, O. Junk, A. Voisard, and J. W. Fluhr, $m R O M A$ - An expert-based approach for the multidisciplinary rating of mHealth applications, IEEE, 2020 IEEE International Conference on Healthcare Informatics (ICHI), pp. 1-11, 2020.

[12] N. J. Lehmann, M.-U. Karagülle, D. Kmiotek, F. Spielmann, B. George, O. Junk, A. Voisard, J. W. Fluhr, L. Ververs, and L. Mielke, mHealthAtlasAn Approach for the Multidisciplinary Evaluation of mHealth Applications, IEEE, 2020 IEEE International Conference on E-health Networking, Application \& Services (Healthcom), pp. 1-5, 2021.

[13] N. J. Lehmann, M.-U. Karagülle, F. Spielmann, B. George, B. Zick, J. Heuer, E. Taegener, A. A. Fahed, A. Voisard, and J. W. Fluhr, mHealthAtlas - An expert-based multi-sided platform for the evaluation of mHealth applications, IEEE, 2021 IEEE International Conference on Healthcare Informatics (ICHI), 2021. (in press)
[14] Weisse Liste gemeinnützige GmbH (Bertelsmann Stiftung), Weisse Liste, https://www.weisse-liste.de/, 2021. (Online; Accessed September 21, 2021) (in German)

[15] Zentralinstitut für die kassenärztliche Versorgung in der Bundesrepublik Deutschland Stiftung des bürgerlichen Rechts, KVAppRadar, https://www.kvappradar.de/, 2021. (Online; Accessed September 21, 2021) (in German)

[16] ORCHA Healthcare Ltd, ORCHA, https://orchahealth.com/, 2021. (Online; Accessed September 21, 2021)

[17] S. Bergauer, The evaluation of processes within the framework of process management (Die Bewertung von Prozessen im Rahmen eines Prozessmanagements), Otto von Guericke Universität Magdeburg, Diploma Thesis, 2009. (in German)

[18] M. Gersch, M. Hewing, and B. Schöler, Business Process Blueprinting - an enhanced view on process performance, Emerald Group Publishing Limited, Business Process Management Journal, vol. 17, num. 5, pp. 732-747, 2011.

[19] J. Richardson, Cost utility analysis: What should be measured?, Elsevier, Social Science \& Medicine, vol. 39, num. 1, pp. 7-21, 1994.

[20] P. Weirich, Decision Space: Multidimensional Utility Analysis, Cambridge University Press, Cambridge Studies in Probability, Induction and Decision Theory, 2001.

[21] T. Ley, M. Jurisch, P. Wolf, and H. Krcmar, Criteria for the performance assessment of processes: A State-of-the-Art (Kriterien zur Leistungsbeurteilung von Prozessen: Ein State-of-the-Art), Institut für Wirtschaftsinformatik, Multikonferenz Wirtschaftsinformatik 2012: Tagungsband der MKWI 2012, 2012. (in German)

[22] M. Bradway, C. Carrion, B. Vallespin, O. Saadatfard, E. Puigdomènech, M. Espallargues, and A. Kotzeva, mHealth Assessment: Conceptualization of a Global Framework, JMIR Publications, JMIR mHealth and uHealth, vol. 5, num. 5, p. e60, 2017.

[23] R. J. M. M. Does, J. van de Heuvel, J. de Mast, and G. C. Niemeijer, Improving quality in healthcare while reducing costs, American Society for Quality, The quality management forum ASQ, pp. 12-15, 2010.

[24] E. J. S. Hovenga, Importance of achieving semantic interoperability for national health information systems, Texto \& Contexto-Enfermagem, vol. 17, num. 1, pp. 158-167, 2008.

[25] GKV-Spitzenverband, Position paper of the National Association of Statutory Health Insurance Funds: Requirements and criteria for Digital Health Applications (Positionspapier des GKV-Spitzenverbandes: Anforderungen und Kriterien an Digitale Gesundheitsanwendungen), 2021. (in German)

[26] L. Stegemann and M. Gersch, Interoperability Technical or economic challenge?, De Gruyter Oldenbourg, it - Information Technology, vol. 61, num. 5-6, pp. 243-252, 2019. 